\title{
Progress in the application and mechanism of metformin in treating non-small cell lung cancer (Review)
}

\author{
CHAN LI* ${ }^{*}$ YANG XUE* ${ }^{*}$ YU-RONG XI ${ }^{*}$ and KE XIE \\ Department of Oncology, Sichuan Academy of Medical Sciences and \\ Sichuan Provincial People's Hospital, Chengdu, Sichuan 610072, P.R. China \\ Received August 18, 2015; Accepted January 4, 2017
}

DOI: $10.3892 / \mathrm{ol} .2017 .5862$

\begin{abstract}
At present, the incidence and mortality of lung cancer demonstrate an increasing trend. Non-small cell lung cancer (NSCLC) accounts for $\sim 80-85 \%$ of all lung cancer cases. Therefore, developing novel and more effective treatments is of great importance. The use of combination therapies, where several anticancer agents are used together, is a promising strategy. Recent studies demonstrate that metformin, which has been utilized for treating diabetes mellitus for $>50$ years, has antitumor effects in numerous types of cancer including NSCLC. Its antitumor effects can be direct and indirect, and it is able to synergize with other physical therapies including targeted anticancer therapy, chemotherapy and radiotherapy. The present review discusses how metformin affects cellular energy metabolism in NSCLC, the mechanism of its antitumor action and its synergy with other therapies. Information and analysis are provided in the present review to stimulate further studies on metformin as an adjunct anticancer treatment.
\end{abstract}

\section{Contents}

1. Introduction

2. Glucose metabolism in NSCLC and the effects of metformin

3. Cellular transport of metformin in NSCLC

4. Antitumor mechanism of metformin and its application in NSCLC

5. Use of metformin in the treatment of NSCLC

Correspondence to: Dr Ke Xie, Department of Oncology, Sichuan Academy of Medical Sciences and Sichuan Provincial People's Hospital, 32 West Second Section First Ring Road, Chengdu, Sichuan 610072, P.R. China

E-mail: 840246753@qq.com

*Contributed equally

Key words: biguanide, metformin, non-small-cell lung cancer, mitochondria, glycolysis, targeted therapy, chemotherapy, radiotherapy
6. Safety of metformin as an antitumor adjuvant

7. Future prospects

\section{Introduction}

The importance of developing improved treatments for non-small cell lung cancer (NSCLC) cannot be overestimated. Lung cancer remains one of the major causes of mortality, with incidence on the increase in numerous parts of the world (1); NSCLC is the most common type, accounting for $80-85 \%$ of all lung cancer cases. Targeting the carcinoma with a combination of several drug types and physical methods, including radiotherapy and phototherapy, is proving to be an effective strategy (2).

Metformin is a biguanidine and a hypoglycemic agent. Since its introduction in Europe in 1957, metformin has been used as a drug for lowering elevated blood glucose levels in diabetes mellitus patients (3). In 2005, it was suggested that metformin could reduce the incidence of cancer, making it into the focus of tumor research (4) and potential applications for metformin in oncology were investigated. It was reported that metformin is able to perform its antitumor action by altering neoplastic cellular energy metabolism (5). Since 2013, to the best of our knowledge there have been $>173$ ongoing clinical trials on the use of metformin in cancer (6). Furthermore, in a recent study, Birsoy et al (7) showed a link between the glucose limitation activity of phenformin, another biguanide, and its effects on the metabolic determinants of cancer cell sensitivity. Due to the rapid growth of cancer cells and high consumption of nutrients, particularly glucose, it was observed that glucose concentration is lower in tumors than in normal tissues (7). However, the majority of cancer cells are able to develop and reproduce rapidly in spite of the low-glucose conditions (7). Biguanide drugs, e.g., phenformin and metformin, are inhibitors of mitochondrial oxidative phosphorylation, and this is thought to account for the observed antineoplastic activity of these diabetes drugs (7).

Since 2004, the present authors have been focusing on the antitumor effects of metformin in NSCLC. A multi-center clinical trial confirmed that metformin could improve chemotherapy survival outcomes for diabetes mellitus patients who have NSCLC (8). The present review discusses how metformin affects cellular energy metabolism in NSCLC, the mechanism 
of its antitumor action and its synergy with other therapies. The goal is to investigate the feasibility of adjunct metformin for treating NSCLC and to stimulate further study.

\section{Glucose metabolism in NSCLC and the effects of met- formin}

Glucose metabolism in normal cells includes glycolysis, aerobic oxidation and the pentose phosphate pathway. The first step of glycolysis is the enzymatic conversion of glucose to pyruvate, which is then reduced to lactate. In aerobic oxidation, pyruvate enters the tricarboxylic acid cycle, and it is eventually completely oxidized to water and $\mathrm{CO}_{2}$ with the production of adenosine triphosphate (ATP) (Fig. 1) (9).

Compared with aerobic oxidation, glycolysis provides less energy per mole of glucose, but it operates at a faster rate. Even under aerobic conditions, cancer cells preferentially utilize glycolysis as their main energy source rather than oxidative phosphorylation and this is known as the 'Warburg effect' (10).

Under normoxic conditions, adenocarcinomas perform glycolysis, whilst squamous cell carcinomas, which are subjected to varying degrees of hypoxia, perform a high level of glycolysis even in anaerobic environments to obtain sufficient energy for survival (11). These findings prompted further studies into how this finding can be utilized in developing new treatments. In particular, the oral antidiabetic drug metformin is able to stimulate glycolysis by altering the activity of specific metabolic enzymes, including fructose-2, 6-bisphosphate (9); therefore, it may promote the switch to glycolysis in NSCLC cells as the main method of producing energy. On the surface, this may have a stimulatory effect on the growth of NSCLC cells, but in fact the switch in the energy pathway, in the presence of metformin, is associated with its primary antitumor mechanism (Fig. 1) (9). As glycolysis provides less energy per mole of glucose, a decrease in ATP generation results in an increased level of adenosine monophosphate (AMP), which leads to an increase in the ratio of intracellular AMP to ATP and an energy metabolism imbalance. This is one of the mechanisms by which metformin is able to achieve its antineoplastic activity. It is likely to involve the active 5'-AMP-activated protein kinase (AMPK) and its downstream signaling pathways (9).

\section{Cellular transport of metformin in NSCLC}

The antitumor effect of metformin on energy metabolism is dependent on whether metformin can be transported into the mitochondria inside NSCLC cells. It has been observed in tracking experiments that metformin is able to move across the cell plasma membrane $(9,12-15)$ and the mitochondrial membranes $(12,16,17)$.

Transport of metformin across the NSCLC cell membrane. At physiological pH, metformin is positively charged (12), meaning the movement of metformin across NSCLC cell membranes may be mediated by organic cation transporters (OCTs) (9). Organic cation transporter 1 (OCT1) is primarily responsible for metformin uptake in the liver (9). Solute carrier family 22 member 18 (SLC22A18) and OCT1 share certain homology (13). The Homo sapiens gene SLC22A18 is located on chromosome 11 at $11 \mathrm{p} 15.5$ (13). It has been demonstrated that microRNA-137 significantly inhibits NSCLC cell proliferation, invasion and migration, as it targets SLC22A18 (14). Provided that SLC22A18 is highly expressed in the NSCLC tissue, it may actively transport metformin into the NSCLC cells, particularly in squamous cell carcinoma and adenocarcinoma. An examination of NSCLC cells has observed that SLC22A18 is primarily expressed in the cell membranes and cytoplasm (13). Additionally, SLC22A18 is not expressed in normal lung tissues, and it is upregulated in squamous cell carcinoma and adenocarcinoma (15).

Transport of metformin across the mitochondrial membrane of NSCLC cells. Mitochondria have both an outer and an inner membrane. Compared with the inner membrane, the permeability of the outer membrane is relatively high (16). Molecules with a molecular mass $\leq 5,000 \mathrm{kDa}$ or less can freely travel through the outer membrane of mitochondria, therefore the environment of the intermembrane space and cytoplasm is similar (16). As metformin is a simple molecule with a molecular mass of only $129 \mathrm{kDa}$, it is able to pass through the outer membrane and enter the intermembrane space freely (16). At physiological $\mathrm{pH}$, metformin exists as a cation (>99.9\%) (12). The mitochondrial membrane potential allows the metformin cation to accumulate in the matrix of the mitochondria (17).

\section{Antitumor mechanism of metformin and its application in NSCLC}

The mitochondrial electron transport chain is composed of complexes I, II, III and IV, all of which have the ability to transfer electrons (18). The protons along the concentration gradient move back across the inner membrane through the enzyme ATP synthase, with the aid of the mitochondrial membrane potential (18). The flow of protons back into the matrix of the mitochondrion via ATP synthase provides sufficient energy for adenosine diphosphate to combine with inorganic phosphate to form ATP (18).

Metformin is thought to target complex I, enabling its antidiabetic and antitumor activity (9). Metformin limits respiration and citric acid cycle activity in the mitochondria and alters cellular bioenergetics (19). A reduction in ATP generation results in increased levels of AMP, which in turn leads to an increase in the ratio of intracellular AMP to ATP, as well as an energy metabolism imbalance (9). This is likely to drive two major signaling pathways: The inhibition of glucagon-induced cyclic AMP synthesis in the liver, and the activation of 5'-AMPK and downstream signaling pathways (9). The antitumour activity of metformin may be direct or indirect (systemic), and its direct effects involve the AMPK signaling pathway (Fig. 2) (9).

The systemic influence is secondary and results in several consequences, including a decrease in body weight, anti-inflammatory actions, improvements in insulin-resistance, and a reduction in systemic levels of glucose and insulin. The direct effects include AMPK-dependent and AMPK-independent signaling pathways (9).

Indirect antitumor effects of metformin in NSCLC: Decreasing body weight. The primary mode of action of metformin in causing body weight loss is via decreasing appetite (20). The 


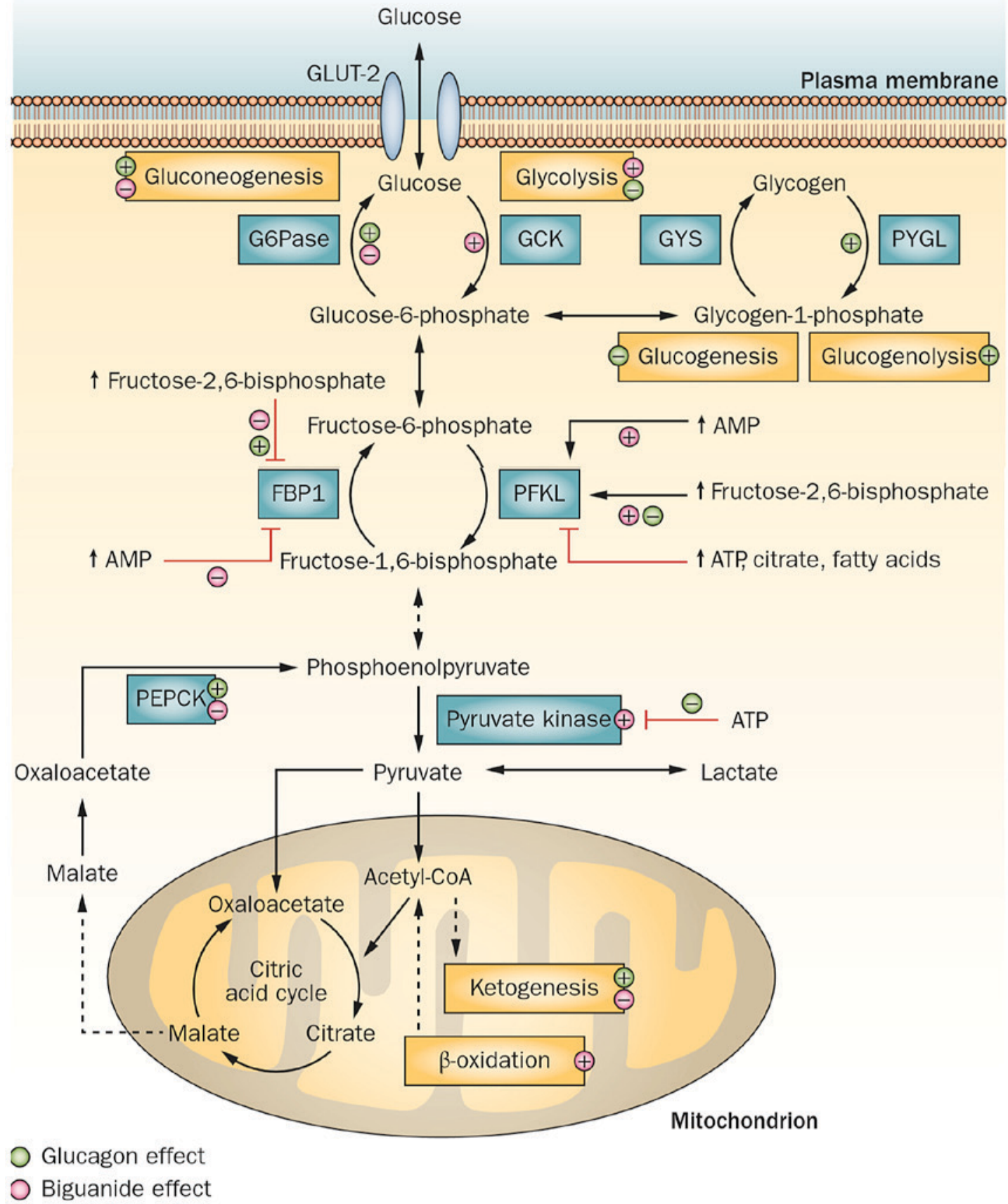

Figure 1. Glucose metabolism (glycolysis and aerobic oxidation) and the hypoglycemic mechanism of biguanides. AMP, adenosine monophosphate; ATP, adenosine triphosphate; FBP1, fructose-bisphosphatase 1; GLUT-2, solute carrier family 2 member 2; G6Pase, glucose-6-phosphatase; GCK, glucokinase; GYS, glycogen synthase 1; PYGL, phosphorylase, glycogen, liver; PFKL, phosphofructokinase, Liver Type; PEPCK, phosphoenolpyruvate carboxykinase 2, mitochondrial. Reprinted by permission from Macmillan Publishers Ltd.: Nature Reviews Endocrinology (9), copyright (2014).

secondary mechanisms include improvements in gastrointestinal physiology and circadian rhythms, and regulation of fat oxidation and storage of fat in liver, skeletal muscle or adipose tissue (20). Dahlberg et al (21) evaluated the association between body-mass index and clinical outcomes for 2,585 NSCLC patients. It was reported that obese patients had improved outcomes earlier in the study compared to normal or overweight patients, but subsequently obesity was demonstrated to increase risk (21). Therefore, for obese NSCLC patients, reducing weight can improve prognosis and metformin may aid in accomplishing this.
Indirect antitumor effects of metformin in NSCLC: Inflammation, tumor progression and immunity. There is increasing evidence that certain tumor-associated inflammatory markers (C-reactive protein, Toll-like receptors 2 and 4, and tumor necrosis factor- $\alpha$ ) are associated with poorer prognosis of certain types of cancer, including NSCLC (22-24). Antitumor immune responses involve CD8+ T lymphocytes and mature dendritic cells (mDCs) (22-28). Alifano et al (25) reported on the role of systemic inflammation, nutritional status and tumor immune microenvironment in determining the outcome 


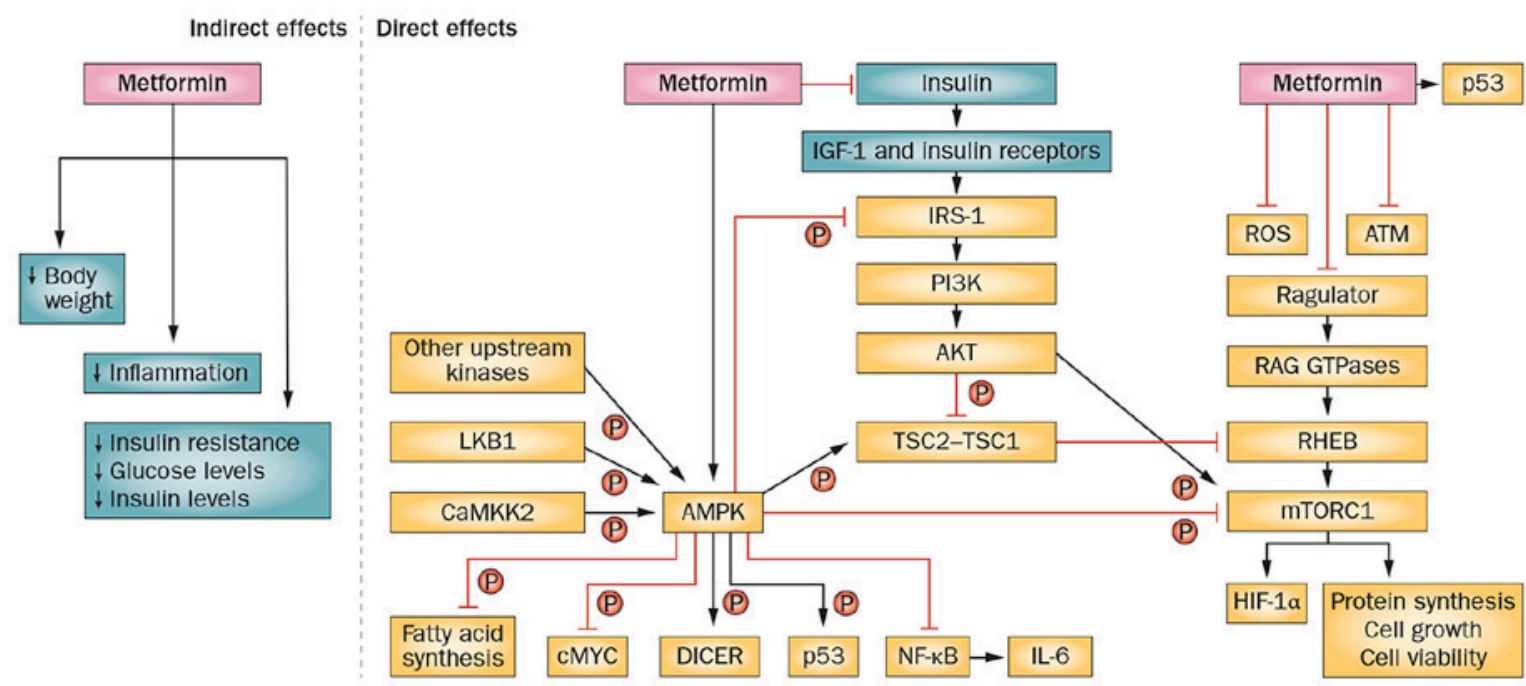

Figure 2. Antitumor mechanisms of metformin. AMPK, adenosine monophosphate-activated protein kinase; ATM, ataxia-telangiectasia mutated; CaMKK2, calcium/calmodulin dependent protein kinase kinase 2; DICER, dicer 1, ribonuclease III; HIF-1 $\alpha$, hypoxia inducible factor 1 alpha subunit; IGF-1, insulin like growth factor 1; IL-6, interleukin-6; IRS-1, insulin receptor substrate 1; LKB1, serine/threonine kinase 11; mTORC1, mechanistic target of rapamycin complex1; NF-кB, nuclear factor-кB; PI3K, phosphoinositide 3-kinase; RHEB, ras homolog enriched in brain; ROS, reactive oxygen species; TSC2-TSC1, tuberous sclerosis 1-tuberous sclerosis 2. Reprinted by permission from Macmillan Publishers Ltd.: Nature Reviews Endocrinology (9), copyright (2014).

of resection in NSCLC patients. It was suggested that the tumoral immune microenvironment is associated with long-term outcome in primary and metastatic tumors in resected NSCLC patients (25).

In NSCLC, a high intratumoral concentration of mDCs and low intratumoral numbers of $\mathrm{CD} 8+\mathrm{T}$ lymphocytes are associated with an improved prognosis (25). The intratumoral concentration of $\mathrm{mDCs}$ is inversely correlated with age. It is lower in males, smokers, and patients with squamous cell carcinoma or chronic obstructive pulmonary disease (25). Therefore, inhibiting inflammation may improve the prognosis of patients with NSCLC. As well as glycemic control, metformin reduces a number of inflammatory markers (C-reactive protein, tumor necrosis factor- $\alpha$, Toll-like receptors 2 and 4) and causes oxidative stress in obese type 2 diabetic patients (24). Furthermore, metformin is able to reduce the production of tumor necrosis factor- $\alpha$ by inhibiting the extracellular signal-regulated kinase-1/2-early-growth response-1 (ERK1/2-Egr-1) signaling pathway in human monocytes (26). Consequently, metformin is able to improve the prognosis of NSCLC.

Indirect antitumor effects of metformin in NSCLC: Improvements in insulin-resistance, and reductions in insulin and glucose levels. An examination of Kirsten rat sarcoma viral oncogene homolog (K-RAS)-induced effects revealed that hyperglycemia is able to promote the expansion of tumor-initiating lung bronchoalveolar stem cells (BASCs) in bronchoalveolar duct junctions (29). The active $K-R A S$ oncogene is able to increase the expression of glucose transporter 1 , thereby promoting glucose uptake and glycolysis in BASCs (29). Hyperglycemia is also able to increase autonomous hyperplasia of BASCs, which leads to oxidative stress and the production of reactive oxygen species (ROS), as well as a reduction in mitochondrial function and inhibition of oxidative phosphorylation (29). Therefore, lung cancer patients may benefit from metformin treatment via the reduction of blood glucose levels.

The ability of metformin to reduce circulating glucose levels may be explained by multiple mechanisms including: i) Reduction in glucose output by inhibiting gluconeogenesis in the liver (9); ii) increase in insulin-mediated glucose uptake in the skeletal muscle by elevating circulating glucagon-like peptide-1 (GLP-1) (30); and iii) increase in expression of GLP-1 receptors in the pancreas $(31,32)$. Metformin indirectly inhibits dipeptidyl peptidase- 4 activity, which reduces the breakdown of GLP-1, thereby increasing levels of circulating GLP-1 (9). Metformin has limited effects on glucose absorption in the digestive tract, and marginally delays the absorption process (9). Metformin also improves insulin resistance by reducing circulating insulin levels (9).

Direct antitumor effects of metformin in NSCLC. Metformin is able to inhibit the mammalian target of rapamycin complex 1 (mTORC1) through either AMP kinase-dependent or independent signaling pathways to achieve antitumor effects (Fig. 2) (9). Metformin may also suppress the phosphorylation of cytokines, including translation initiation factor 4E-binding protein 1 and S6 kinase-1, by inhibiting mTOR. Metformin is able to decrease protein synthesis, tumor cell proliferation and survival (33). Solid tumors often exhibit a hypoxic microenvironment state (34). Hypoxia-inducible factor-1 (HIF-1) is a nuclear transcription factor produced by cells under hypoxic conditions. HIF-1 $\alpha$, a subunit of HIF-1, is responsible for HIF-1 activity and is highly expressed in NSCLC cells. HIF-1 $\alpha$ serves an important role in NSCLC tumorigenesis, local invasion and distant metastasis, by upregulating the expression of the nuclear proliferation protein antigen Ki-67 and vascular endothelial growth factor (VEGF) (34). By inhibiting mTORC1, metformin inhibits HIF- $1 \alpha$ and is able to reduce local invasiveness and metastasis in NSCLC (33). 
Metformin improves the prognosis of NSCLC via the AMPK signaling pathway. Metformin may cause phosphorylation of the tumor suppressor gene tuberous sclerosis 2 by phosphorylating AMPK, which in turn results in the inhibition of the mTORC-1 activator, GTPase and Ras homolog enriched in brain. The liver kinase B-1 (LKB1) gene in Homo sapiens encodes a tumor suppressor that phosphorylates the AMPK $\alpha$ subunit at Thr-172 to activate AMPK (33). Activated AMPK inhibits tumorigenesis by inhibiting mTOR (Fig. 2) (9). The phosphorylation of AMPK may also result in direct phosphorylation and inhibition of the positive regulatory-associated protein of mTOR (Fig. 3) (33). Ultimately, metformin may achieve its primary antitumor effect by inhibiting mTORC1. The incidences of LKB1 mutations in adenocarcinomas and squamous cell carcinomas are reported to be 13 and 5\%, respectively (35). With certain LKB1 gene mutations, the LKB1-AMPK axis remains mostly functional and can be stimulated by metformin in NSCLC cells (33). Metformin could serve an effective role in the treatment of NSCLC via the LKB1-AMPK-mTOR signaling pathway $(33,35-37)$. NSCLC patients with a high level of phosphorylated (p)-AMPK have higher overall survival (OS) and recurrence free survival (RFS) rates, particularly in adenocarcinoma. However, in squamous cell lung cancer, the level of pAMPK does not affect OS and RFS. Taken together, these data support the conclusion that NSCLC patients may benefit from metformin adjunct therapy through its inhibition of mTOR by activating the LKB1/AMPK signaling pathway (38).

Metformin improves NSCLC prognosis via an AMPK-independent signaling pathway. Metformin was reported to target liver tumor-initiating cells through the phosphoinositide 3-kinase (PI3K)/AKT/mTOR survival pathway both in vivo and in vitro (39). The PI3K/AKT/mTOR signaling pathway was aberrantly activated in squamous cell lung carcinoma (40), and the aberrant activation of this signaling pathway is more common in squamous cell lung carcinoma than in adenocarcinoma (41). In addition, in patients with adenocarcinoma and epidermal growth factor receptor (EGFR)-activating mutations, the aberrant activation of the PI3K/AKT/mTOR signaling pathway is one of the mechanisms of acquired resistance to EGFR-tyrosine kinase inhibitors (TKIs) (41). A further study demonstrated that activation of the PI3K/AKT/mTOR signaling pathway in NSCLC leads to a more aggressive form of the disease and a poorer prognosis (42). Inhibition of the $\mathrm{PI} 3 \mathrm{~K} / \mathrm{AKT} / \mathrm{mTOR}$ signaling pathway may overcome radioresistance, chemoresistance and immune evasion in NSCLC (42). Metformin can block the insulin-like growth factor-1-insulin signaling pathway via phosphorylation of insulin receptor substrate 1 (IRS-1), which inhibits the IRS-1/PI3K/AKT signaling pathway to prevent mTOR activation (Fig. 2) (9). Therefore, metformin may provide benefit to NSCLC patients through its inhibition of the PI3K/AKT/mTOR signaling pathway (9).

\section{Use of metformin in the treatment of NSCLC}

Metformin and targeted therapy. The development of targeted therapies for lung cancer has been centered on pharmaceutical interventions to block the EGFR-TKI axis. A number

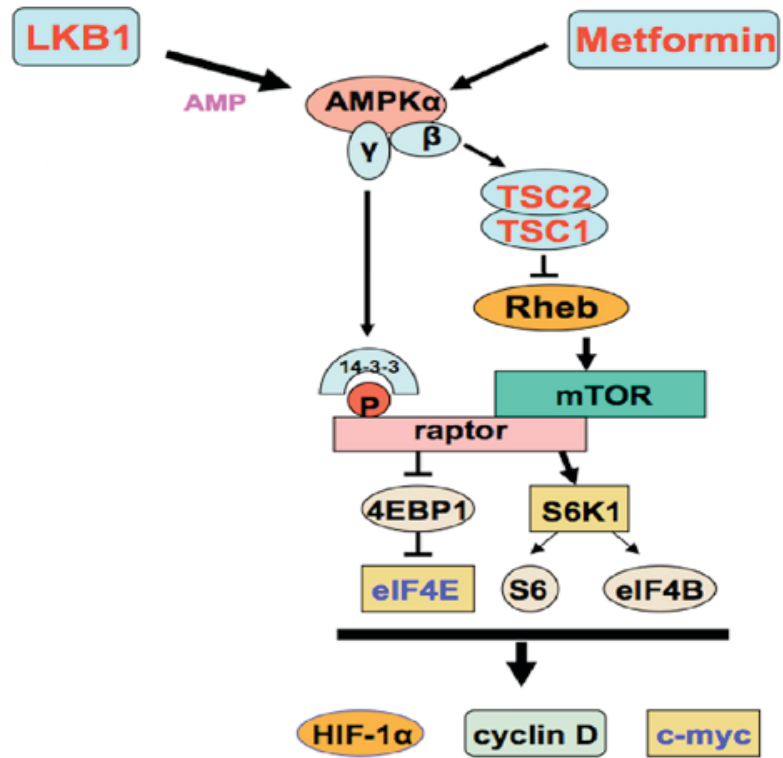

Figure 3. AMPK and its downstream signaling pathways. AMP, adenosine monophosphate; AMPK, adenosine monophosphate-activated protein kinase; elF4E, E74 like ETS transcription factor 4; RAPTOR, regulatory associated protein of MTOR complex 1; RHEB, Ras homolog enriched in brain; S6K1, ribosomal protein S6 kinase B1; TSC1, tuberous sclerosis 1; $\mathrm{TSC} 2$, tuberous sclerosis 2; 4EBP1, eukaryotic translation initiation factor 4E binding protein 1 . Reproduced with permission (33).

of EGFR-TKI inhibitors are currently in use, including gefitinib (Iressa) and erlotinib (Tarceva), which are able to block EGFR-mediated proliferation and anti-apoptosis signaling pathways. However, following 10 months of treatment, these agents may lose their effectiveness due to drug resistance (43). The primary resistance of TKI is associated with $K-R A S$ mutations. The causes of secondary resistance of TKI include: Second-site mutation of the EGFR kinase domain (T790M), other kinase amplifications (such as MET), NSCLC conversion into small cell lung cancer and epithelial-mesenchymal transition (EMT).

As metformin is able to inhibit the PI3K/AKT/mTOR signaling pathway, using metformin in combination with an EGFR-TKI blocker could produce a synergistic, antiproliferative effect on the tumor. As mentioned previously, the aberrant activation of the PI3K/AKT/mTOR signaling pathway is one of the mechanisms by which patients with adenocarcinoma and EGFR-activating mutations are able to gain resistance to EGFR-TKI blockers. Metformin adjunct therapy could reverse EGFR-TKI resistance by inhibiting the PI3K/AKT/mTOR signaling pathway (41). Li et al (43) reported that when metformin was combined with an EGFR-TKI blocker (gefitinib or erlotinib) in vivo and in vitro, the interleukin-6/signal transducer and activator of transcription 3 (IL-6/STAT3) signaling pathway was inhibited, which reversed EMT and eventually overcame resistance in NSCLC cells. In 2013, one clinical trial involving combined metformin and gefitinib to treat NSCLC patients resulted in one-year progression-free survival (6).

Sorafenib is a novel, multi-target anticancer drug that inhibits a number of kinases, including AMPK (44). Sorafenib has dual antitumor effects. When it is used in combination with metformin to treat NSCLC, the antitumor AMPK 
signaling pathway is activated through either calcium/calmodulin-dependent-kinase-kinase-2 or LKB1. Sorafenib can also cause cytosolic calcium mobilization and mitochondrial calcium overload. Therefore, it can promote mitochondrial ROS production and lead to tumor cell death (44). Notably, the average tumor volume and growth rate were lower in patients treated with the combination therapy vs. patients treated with sorafenib alone (44).

Metformin and radiotherapy. Radiotherapy is a method of destroying tumors via the biological effects of ionizing radiation (45). The ataxia-telangiectasia mutated (ATM) gene product is part of a signal transduction cascade that is important for repairing damaged DNA and, as a consequence when mutated may lead to sensitivity to ionizing radiation. Therefore, the degree of radiosensitivity or radioresistance of NSCLC cells is dependent on ATM. Lung cancer radiotherapy can achieve antitumor effects by causing G2-M arrest and cytotoxicity, by activating the ATM-AMPK-tumor protein $\mathrm{p} 53$ (p53)/cyclin dependent kinase inhibitor 1A (p21 ${ }^{\text {cipl}}$ ) signaling pathway, reducing the phosphorylation of AKT and inhibiting AKT-mTOR-eukaryotic translation initiation factor $4 \mathrm{e}$ binding protein 1 (4EBP1) (46). Resistance to radiation is associated with the AMPK and AKT-mTOR signaling pathways. Metformin is able to activate AMPK and inhibit AKT-mTOR to sensitize lung tumors to ionizing radiation (9).

Storozhuk et al (47) tested the hypothesis that metformin can inhibit growth and enhance radiosensitivity of NSCLC through ATM and AMPK. It was reported that combined treatment with metformin and radiotherapy consistently activated the ATM-AMPK-p53/p21 ${ }^{\text {cipl }}$ signaling pathway and inhibited the AKT-mTOR-4EBP1 signaling pathway (47). A constant concentration of $7.8 \mu \mathrm{M}$ metformin was achieved in patients who took a daily dosage of $850-1,700 \mathrm{mg}$. As a daily dosage of 2.5-3.0 g of metformin has no notable toxicity, a recommended dosage of $850-1,700 \mathrm{mg}$ metformin is well within the safe range (47). Since 2013, to the best of our knowledge there have been three studies involving the use of metformin in combination with radiotherapy to treat NSCLC (48-50). These trials led to progression-free survival according to Response Evaluation Criteria in Solid Tumors (51).

Metformin and chemotherapy. Cisplatin is the most commonly used chemotherapy drug for the treatment of NSCLC, and it has been used as the first-line treatment for NSCLC in patients without EGFR mutations (52). Chemoresistance to cisplatin is associated with ROS production, IL-6 secretion and STAT3 phosphorylation. In NSCLC, STAT3 is active and may facilitate tumor proliferation, survival and angiogenesis by overexpressing anti-apoptotic proteins (Bcl-2-like protein 1 and myeloid cell leukemia 1), cell cycle regulators (cyclin D1 and c-Myc), and VEGF (52). Cisplatin is able to promote the generation of ROS and the phosphorylation of STAT3 (52).

Metformin is able to inhibit cisplatin-induced ROS generation, STAT3 phosphorylation and autocrine IL-6 secretion, thereby enhancing the chemosensitivity of NSCLC to cisplatin (52). Furthermore, metformin is able to improve the effect of cisplatin in A549/CDDP cells (53). Metformin also has a synergistic effect with cisplatin or etoposide in large cell lung carcinoma cells (NCI-H460) by increasing the antitumor effectiveness of the chemotherapeutics (54). There are several clinical trials underway to examine the effectiveness of the combination of cisplatin or carboplatin with metformin. Using a combination of cisplatin or carboplatin with metformin may be a promising treatment for NSCLC patients $(49,50,55,56)$.

\section{Safety of metformin as an antitumor adjuvant}

The safety of metformin has been previously investigated for the treatment of type 2 diabetes mellitus. A number of major adverse effects have been reported concerning gastrointestinal reactions, including nausea, abdominal discomfort and diarrhea. These symptoms are usually mild and can be treated (57). At present, large-scale clinical trials and meta-analyses have yet to show evidence that metformin increases the risk of lactic acidosis (57). Metformin has no reported renal toxicity, but it is excreted by the kidneys (57). This means metformin may accumulate in patients with renal impairments, thus increasing the risk of lactic acidosis. However, metformin-induced liver toxicity is rare (57). Considering that metformin has been in use for $>50$ years and its side-effects (including low incidence of hypoglycemia and transient gastrointestinal reactions) have been thoroughly investigated and that the benefits outweigh the side effects, the conclusion is that it is safe for use in clinical trials for the treatment of cancer (57).

\section{Future prospects}

NSCLC is the most commonly occurring solid tumor worldwide (1). Although progress in improving chemotherapy, radiotherapy and targeted therapies has increased patient survival, the five-year overall survival time remains low (1). Cisplatin one of the most commonly used first-line chemotherapeutic agents for NSCLC (52), has several toxic side effects. The aim is to find novel adjuvant drugs, in order to enhance the antitumor effect of cisplatin, but without increasing the dose and toxicity. There is compelling evidence from a number of preclinical studies to suggest that the antidiabetic drug metformin is a prime candidate as an adjunct to chemotherapy and radiotherapy in the treatment of NSCLC $(45-47,52-54,58)$. It has been demonstrated that metformin is able to improve survival time among diabetes mellitus patients with stage IV NSCLC (59). Furthermore, numerous NSCLC patients in advanced stages are too weak to undergo chemotherapy, radiotherapy or surgery, thus reducing their chance of survival. Combining metformin with such treatments, may be a promising strategy to improve patient survival.

Squamous cell lung carcinoma is insensitive to chemotherapy, radiotherapy and targeted therapy (40). For the majority of patients, it is too late to undergo surgical resection by the time they are diagnosed. The US Food and Drug Administration has approved nivolumab and ramucirumab for the treatment of NSCLC, but improvements in overall survival and recurrence rates have yet to be reported. As described previously, the PI3K/AKT/mTOR signaling pathway is aberrantly activated in squamous cell lung carcinoma (40), which has been correlated with poor prognosis for these patients (42). As metformin is able to inhibit the PI3K/AKT/mTOR signaling pathway (42), it may improve survival outcomes when used 
in combination with everolimus or gemcitabine and cisplatin chemotherapy.

At present, to the best of our knowledge there are nine clinical trials involving the use of metformin in treating NSCLC. The potential for the long-term use of metformin in improving survival and recurrence is yet to be evaluated.

\section{References}

1. Siegel RL, Miller KD and Jemal A: Cancer Statistics, 2017. CA Cancer J Clin 67: 7-30, 2017.

2. Rodin D, Grover S, Xu MJ, Hanna TP, Olson R, Schreiner LJ, Munshi A, Mornex F, Palma D, Gaspar LE, et al: Radiotherapeutic management of non-small cell lung cancer in the minimal resource setting. J Thorac Oncol 11: 21-29, 2016.

3. Mu YM, Ji LN, Ning G, Li GW, Li Y, Sun ZL, Li YB, Zhao JJ, Wang WQ, Zhu DL, et al: Chinese experts consensus statement on metformin in the clinical practice. Chin J Diabetes 24: 871-884, 2016 (In Chinese).

4. Evans JM, Donnelly LA, Emslie-Smith AM, Alessi DR and Morris AD: Metformin and reduced risk of cancer in diabetic patients. BMJ 330: 1304-1305, 2005.

5. Pollak M: Potential applications for biguanides in oncology. J Clin Invest 123: 3693-3700, 2013.

6. U.S. National Library of Medicine, ClinicalTrials gov: Metformin and cancer. http://www.clinicaltrials.gov/ct2/results?term=metfo rmin+and+cancer\&Search=Search. Accessed January 7, 2014.

7. Birsoy K, Possemato R, Lorbeer FK, Bayraktar EC, Thiru P, Yucel B, Wang T, Chen WW, Clish CB and Sabatini DM: Metabolic determinants of cancer cell sensitivity to glucose limitation and biguanides. Nature 508: 108-112, 2014.

8. Tan BX, Yao WX, Ge J, Peng XC, Du XB, Zhang R, Yao B, Xie K, Li LH, Dong H, et al: Prognostic influence of metformin as first-line chemotherapy for advanced nonsmall cell lung cancer in patients with type 2 diabetes. Cancer 117: 5103-5111, 2011.

9. Pernicova I and Korbonits M: Metformin-mode of action and clinical implications for diabetes and cancer. Nat Rev Endocrinol 10: 143-156, 2014.

10. Warburg O: Metabolism of tumours. Biochem Z 142: 317-333, 1923.

11. Schuurbiers OC, MeijerTW, Kaanders JH,Looijen-Salamon MG, de Geus-Oei LF, van der Drift MA, van der Heijden EH, Oyen WJ, Visser EP, Span PN and Bussink J: Glucose Metabolism in NSCLC is histology-specific and diverges the prognostic potential of 18FDG-PET for adenocarcinoma and squamous cell carcinoma. J Thorac Oncol 9: 1485-1493, 2014

12. Graham GG, Punt J, Arora M, Day RO, Doogue MP, Duong JK, Furlong TJ, Greenfield JR, Greenup LC, Kirkpatrick CM, et al: Clinical pharmacokinetics of metformin. Clin Pharmacokinet 50: 81-98, 2011.

13. Lei M, Cheng Q, Zhao Y, Liu T, Wang X, Deng Y, Yang J and Zhang Z: Expression and its clinical significance of SLC22A18 in non-small cell lung cancer. Zhongguo Fei Ai Za Zhi 15: 17-20, 2012 (In Chinese).

14. Zhang B, Liu T, Wu T, Wang Z, Rao Z and Gao J: microRNA-137 functions as a tumor suppressor in human non-small cell lung cancer by targeting SLC22A18. Int J Biol Macromol 74: 111-118, 2015.

15. Lei M, Cheng QS, Zhang ZP, Li XF, Huang Q and Zhang ZP: Expression of SLC22A18 in NSCLC and its correlation with chemoresistance of NSCLC. Chin J Cancer Pre Treat 21: 368-371, 2014 (In Chinese).

16. Alberts B, Johnson A, Lewis J, Raff M, Roberts K and Walter P: Molecular Biology of the Cell. 4th edition. Garland Science, New York, NY, 2002.

17. Wheaton WW, Weinberg SE, Hamanaka RB, Soberanes S, Sullivan LB, Anso E, Glasauer A, Dufour E, Mutlu GM, Budigner GS and Chandel NS: Metformin inhibits mitochondrial complex I of cancer cells to reduce tumorigenesis. Elife 3: e02242, 2014.

18. Mitchell P: Coupling of phosphorylation to electron and hydrogen transfer by a chemi-osmotic type of mechanism. Nature 191: $144-148,1961$

19. Andrzejewski S, Gravel SP, Pollak M and St-Pierre J: Metformin directly acts on mitochondria to alter cellular bioenergetics. Cancer Metab 2: 12, 2014.
20. Malin SK and Kashyap SR: Effects of metformin on weight loss: Potential mechanisms. Curr Opin Endocrinol Diabetes Obes 21: 323-329, 2014.

21. Dahlberg SE, Schiller JH, Bonomi PB, Sandler AB, Brahmer JR, Ramalingam SS and Johnson DH: Body mass index and its association with clinical outcomes for advanced non-small-cell lung cancer patients enrolled on Eastern Cooperative Oncology Group clinical trails. J Thorac Oncol 8: 1121-1127, 2013.

22. Alifano M, Falcoz PE, Seegers V, Roche N, Schussier O, Younes M, Antonacci F, Forgez P, Dechartres A, Massard G, et al: Preresection serum C-reactive protein measurement and survival among patients with resectable non-small cell lung cancer. J Thorac Cardiovasc Surg 142: 1161-1167, 2011

23. Li D, Jin Y, Sun Y, Lei J and Liu C: Knockdown of toll-like receptor 4 inhibits human NSCLC cancer cell growth and inflammation cytokine secretion in vitro and in vivo. Int J Oncol 45: 813-821, 2014.

24. Andrews M, Soto N and Arredondo M: Effect of metformin on the expression of tumor necrosis factor- $\alpha$, Toll like receptors $2 / 4$ and $\mathrm{C}$ reactive protein in obese type-2 diabetic patients. Rev Med Chil 140: 1377-1382, 2012 (In Spanish).

25. Alifano M, Mansuet-Lupo A, Lococo F, Roche N, Bobbio A, Canny E, Schussler O, Dermine H, Régnard JF, Burroni B, et al: Systemic inflammation, nutritional status and tumor immune microenvironment determine outcome of resected non-small cell lung cancer. PLoS One 9: e106914, 2014.

26. Arai M, Uchiba M, Komura H, Mizuochi Y, Harada N and Okajima K: Metformin, an antidiabetic agent, suppresses the production of tumor necrosis factor and tissue factor by inhibiting early growth response factor-1 expression in human monocytes in vitro. J Pharmacol Exp Ther 334: 206-213, 2010.

27. Pearce EL, Walsh MC, Cejas PJ, Harms GM, Shen H, Wang LS, Jones RG and Choi Y: Enhancing CD8 T-cell memory by modulating fatty acid metabolism. Nature 460: 103-107, 2009.

28. Araki K and Ahmed R: AMPK: A metabolic switch for CD8+ T-cell memory. Eur J Immunol 43: 878-881, 2013.

29. Micucci C, Orciari S and Catalano A: Hyperglycemia promotes K-Ras-induced lung tumorigenesis through BASCs amplification. PLoS One 9: e105550, 2014.

30. Sjoberg KA, Rattigan S, Jeppesen JF, Lundsgaard AM, Holst JJ and Kiens B: Differential effects of glucagon-like peptide-1 on microvascular recruitment and glucose metabolism in short-and long-term insulin resistance. J Physiol 593: 2185-2198, 2015.

31. Maida A, Lamont BJ, Cao X and Drucker DJ: Metformin regulates the incretin receptor axis via a pathway dependent on peroxisome proliferator-activated receptor- $\alpha$ in mice. Diabetologia 54: 339-349, 2011.

32. Lenhard JM, Croom DK and Minnick DT: Reduced serum dipeptidyl peptidase-IV after metformin and pioglitazone treatments. Biochem Biophys Res Commun 324: 92-97, 2004.

33. Han D, Li S J, Zhu YT, Liu L and Li MX: LKB1/AMPK/mTOR signaling pathway in non-small-cell lung cancer. Asian Pac J cancer Prev 14: 4033-4039, 2013.

34. Xu HY, Zhong H, Lu SW, Li J and Zhao H: Correlation of expression of HIF-1 $\alpha$ to proliferation and neovascularization of tumor and prognosis of patients with non-small cell lung cancer. Journal of Clinical Pulmonary Medicine 19: 73-76, 2014 (In Chinese)

35. Koivunen JP, Kim J, Lee J, Rogers AM, Park JO, Zhao X, Naoki K, Okamoto I, Nakagawa K, Yeap BY, et al: Mutation in LKB1 tumor suppressor are frequently detected in tumours from Caucasian but not Asia lung cancer patients. Br J Cancer 99: 245-252, 2008

36. Dong LX, Sun LL, Zhang X, Pan L, Lian LJ, Chen Z and Zhong DS: Negative regulation of mTOR activity by LKB1-AMPK signaling in non-small cell lung cancer cells. Acta Pharmacol Sin 34: 314-318, 2013.

37. Marcus AI and Zhou W: LKB1 regulated pathways in lung cancer invasion and metastasis. J Thorac Oncol 5: 1883-1886, 2010.

38. William WN, Kim JS, Liu DD, Solis L, Behrens C, Lee JJ, Lippman SM, Kim ES, Hong WK, Wistuba II, et al: The impact of phosphorylated AMP-activated protein kinase expression on lung cancer survival. Ann Oncol 23: 78-85, 2012.

39. Lin F, Yan W, Song G, Ting W, Hu T and Wu G: Metformin targets liver tumor-initiating cells through the PI3K/Akt/mTOR survival pathway. Chin Sci Bull 59: 3585-3594, 2014.

40. Beck JT, Ismail A and Tolomeo C: Targeting the phosphatidylinositol 3-kinase (PI3K)/AKT/mammalian target of rapamycin (mTOR) pathway: An emerging treatment strategy for squamous cell lung carcinoma. Cancer Treat Rev 40: 980-989, 2014. 
41. Fumarola C, Bonelli MA, Petronini PG and Alfieri RR: Targeting $\mathrm{PI} 3 \mathrm{~K} / \mathrm{AKT} / \mathrm{mTOR}$ pathway in non small cell cancer. Biochem Pharmacol 90: 197-207, 2014.

42. Heavey S, O'Byrne KJ and Gately K: Strategies for co-targeting the PI3K/AKT/mTOR pathway in NSCLC. Cancer Treat Rev 40: 445-456, 2014

43. Li L, Han R, Xiao H, Lin C, Wang Y, Liu H, Li K, Chen H, Sun F, Yang Z, et al: Metformin sensitizes EGFR-TKI-resistant human lung cancer cells in vitro and in vivo through inhibition of IL-6 signaling and EMT reversal. Clin Cancer Res 20: 2714-2726, 2014

44. Groenendijk FH, Mellema WW, van der Burg E, Schut E, Hauptmann M, Horlings HM, Willems SM, van den Heuvel MM, Jonkers J, Smit EF and Bernards R: Sorafenib synergizes with metformin in NSCLC through AMPK pathway activation. Int J Cancer 136: 1434-1444, 2015.

45. Kim BM, Hong Y, Lee S, Liu P, Lim JH, Lee YH, Lee TH, Chang KT and Hong Y: Therapeutic implications for overcoming radiation resistance in cancer therapy. Int J Mol Sci 16: 26880-26913, 2015

46. Storozhuk Y, Sanli T, Hopmans SN, Schultz C, Farrell T, Cutz JC, Steinberg GR, Wright J, Singh G and Tsakiridis T: Chronic modulation of AMP-Kinase, Akt and mTOR pathways by ionizing radiation in human lung cancer xenografts. Radiat Oncol 7: 71, 2012.

47. Storozhuk Y, Hopmans SN, Sanli T, Barron C, Tsiani E, Cutz JC, Pond G, Wright J, Singh G and Tsakiridis T: Metformin inhibits growth and enhances radiation response of non-small cell lung cancer (NSCLC) through ATM and AMPK. Br J Cancer 108 2021-2032, 2013.

48. Heath Skinner: Metformin in Non small Cell Lung Cancer (NSCLC). ClinicalTrials.gov. https://www.clinicaltrials . gov/ct2/show/NCT02285855. Accessed November 5, 2014.

49. Theodoros Tsakiridis: Chemotherapy and Radiation Therapy With or Without Metformin Hydrochloride in Treating Patients With Stage III Non-small Cell Lung Cancer. ClinicalTrials. gov. https://www.clinicaltrials.gov/ct2/show/NCT02186847. Accessed July 8, 2014.

50. Theodoros Tsakiridis: Advanced Lung Cancer Treatment With Metformin and Chemo-Radiotherapy (ALMERA). ClinicalTrials. gov. https://www.clinicaltrials.gov/ct2/show/NCT02115464. Accessed April 14, 2014.
51. Wolchok JD, Hoos A, O'Day S, Weber JS, Hamid O, Lebbé C, Maio M, Binder M, Bohnsack O, Nichol G, et al: Guidelines for the evaluation of immune therapy activity in solid tumors: Immune-related response criteria. Clin Cancer Res 15: 7412-7420, 2009.

52. Lin CC, Yeh HH, Huang WL, Yan JJ, Lai WW, Su WP, Chen HH and Su WC: Metformin enhances cisplatin cytotoxicity by suppressing signal transducer and activator of transcription-3 activity independently of the liver kinase B1-AMP-activated protein kinase pathway. Am J Respir cell Mol Biol 49: 241-250, 2013.

53. Wang $\mathrm{Y}$, Lin $\mathrm{B}$, Wu J, Zhang $\mathrm{H}$ and $\mathrm{Wu} \mathrm{B}$ : Metformin inhibits the proliferation of A549/CDDP cells by activating P38 mitogen-activated protein kinase. Oncol Lett 8: 1269-1274, 2014.

54. Teixeira SF, Guimarães Idos S, Madeira KP, Daltoé RD, Silva IV and Rangel LB: Metformin synergistically enhances antiproliferative effects of cisplatin and etoposide in NCI-H460 human lung cancer cells. J Bras Pneumol 39: 644-649, 2013.

55. Benjamin Levy: Metformin and Carbohydrate Restriction With Platinum Based Chemotherapy In Stage IIIB/IV Non-Squamous Non-small Cell Lung Cancer (NS-NSCLC) (METRO). ClinicalTrials.gov. https://www.clinicaltrials. gov/ct2/show/NCT02019979. Accessed December 13, 2013.

56. Yumin Yeh: Metformin in Stage IV Lung Adenocarcinoma. ClinicalTrials.gov. https://www.clinicaltrials. gov/ct2/show/NCT01997775. Accessed November 17, 2013.

57. Li M and Ji LN: The safety of Metformin in Type 2 diabetes. Chin J Diabetes 22: 289-292, 2014 (In Chinese).

58. Morgillo F, Sasso FC, Della Corte CM, Festino L, Manzo A, Martinelli E, Troiani T, Capuano A and Ciardiello F: Metformin in lung cancer: Rationale for a combination therapy. Expert Opin Investig Drugs 22: 1401-1409, 2013.

59. Lin JJ, Gallagher EJ, Sigel K, Mhango G, Galsky MD, Smith CB, LeRoith D and Wisnivesky JP: Survival of patients with stage IV lung cancer with diabetes treated with metformin. Am J Respir Crit Care Med 191: 448-454, 2015. 